\title{
Importance of Perls' Stain as A Routine Test in Anaemia in Adults
}

\author{
Riju Rani Deka ${ }^{1 *}$, Deba Kumar Saikia ${ }^{2}$, Sanjeeb Kakati ${ }^{3}$ and Bipul kumar Das ${ }^{4}$ \\ ${ }^{1}$ Department of Pathology, Tezpur Medical College, Tezpur, Assam,India \\ ${ }^{2}$ Department of Pathology, Gauhati Medical College, Guwahati, Assam India \\ ${ }^{3}$ Department of Medicine, Assam Medical College, Barbari, Dibrugarh, Assam India \\ ${ }^{4}$ Department of Paediatrics, Gauhati Medical College, Guwahati, Assam India
}

\begin{abstract}
Background: Anaemia is one of the frequent finding in patients of all age. Proper diagnosis is of utmost important to avoid fatal morbidity in untreated chronic cases. Aims: This study is meant to observe the role of Perls' stain in routine practice in etiological evaluation of anaemia in adults.

Methods: Prospective cross sectional study from June 2007 to July 2010. 128 cases of anaemia, alone or as a part of bicytopenia and pancytopenia, in 20-80 years of age, who were recommended by clinicians for bone marrow aspiration analysis after initial routine haematological investigation, were collected as samples. Perls' stain was done on bone marrow smears to estimate marrow iron store in fragments and ring sideroblasts in all the smears.

Result: Anaemia was most common haematological presentation(49\%), mainly in males (62\%) and majority in $20-30$ years of age (31.3\%). Iron deficiency anaemia was most common cause of anaemia(59.6\%) with 0 to1+ iron store. Megaloblastic anaemia, aplastic anaemia, thalassemia and other haemoglobinnopathies, anaemia of chronic disease(AOCD), myelodysplastic syndrome(MDS) and secondary sideroblastic anaemia(SA) were other causes of anaemia found. $3.2 \%$ cases had ring sideroblasts of which $0.78 \%$ was MDS and $2.3 \%$ was secondary SA.

Conclusion: Perls' stain is a cheap and relatively simple test that can be used as a routine test in all bone marrow aspirates to provide provisional diagnosis of some relatively infrequent causes of anaemias which otherwise could be missed when only non invasive methods are relied upon for assessing body iron content.
\end{abstract}

Keywords: Anaemia in Adults, Bone Marrow Iron Store, Myelodysplastic Syndrome, Perls' Stain, Sideroblastic Anaemia

\section{Introduction}

Clinicians frequently identify anaemia in the older patients but data on the prevalence in this part of the country are unavailable. According to WHO worldwide prevalence of anaemia to be $24.8 \% .^{[1]}$ The common causes of anaemia in adults are anaemia of chronic disease(AOCD), iron deficiency anaemia(IDA) due to gastrointestinal bleeding, $\mathrm{B}_{12}$ deficiency anaemia and myelodysplastic syndrome(MDS).Identification of the causes can decrease the morbidity of the patients and hence should be diagnosed at an earliest.

Usually clinical and non-invasive haematological investigations can diagnose most of the anaemia but few conditions require demonstration of ring sideroblasts under light microscope as one of the criteria for diagnosis. Again, AOCD may complicate the interpretation of hematinic markers when IDA coexist. ${ }^{[2]}$ Therefore the gold standard for diagnosis of IDA is to asses bone marrow iron status by Perls' stain. ${ }^{[3]}$ A study found bone marrow iron staining to be more reliable to exclude IDA rather than its diagnosis. ${ }^{[4]}$
The study here is to find out the prevalence of these conditions in the adult patients presenting with anaemia and hence the role of Perls' stain as a routine test in evaluation of bone marrow aspirates in cases of anaemia in adults.

\section{Materials and Methods}

IThis prospective pathological study was conducted in the Department of Pathology, Assam Medical College and Hospital, Dibrugarh for 3 year from June 2007 to July 2010. Anemia was defined according to World Health Organization (WHO) criteria as a hemoglobin concentration below $12 \mathrm{~g} / \mathrm{dL}$ in women and below $13 \mathrm{~g} /$ $\mathrm{dL}$ in men Irrespective of sex, all cases of anaemia alone or as a part of bicytopenia and pancytopenia, in an age of 20-80 years, who were recommended by clinicians for bone marrow aspiration analysis after initial routine haematological investigation were collected as samples.

\section{Exclusion Criteria:}

1. Diagnosed case of anaemia in pregnant women.

2. Patients diagnosed to have malignancy of any kind. 


\section{Patients receiving chemotherapy or radiotherapy.}

Sample Size: A total of 136 cases were recommended for bone marrow evaluation of which 128 cases could be evaluated. 5 samples were highly hemodiluted for evaluation while 3 cases showed dry tape.

In all the cases, samples were collected in EDTA vial with proper anticoagulant ratio. Samples for Prothrombin time were collected in $32 \mathrm{gm} / 1$ trisodium citrate anticoagulant solution in proper ratio corrected for haematocrit. Reticulocyte count was done manually using $1 \%$ new methylene blue solution. Complete blood count was done in sysmax $800 \mathrm{i}$ cell counter quality control of which was maintained daily. Peripheral blood smears were stained with Leishmen's stain.

Written consent from the patients after explaining the procedure was taken. Under all aseptic and antiseptic conditions, bone marrow aspirations were done from posterior superior iliac spine, $0.2-0.5 \mathrm{ml}$ of samples were collected, smears were made to get minimum 7-10 particles for assessment. MGG staining was used for morphological evaluation of bone marrow aspirates. Perls' stain with working pararosalinine solution as counter stain was used.

Diagnosis of iron deficiency anaemia was made on the basis of PBS finding of microcytic hypochromic anaemia and mild to severe poikilocytosis, micronormoblastic erythropoiesis and absent iron store in marrow fragments. Diagnosis of megaloblastic anaemia was based on history, anaemia to pancytpenia, macrocytosis and hypersegmented neutrophils in PBS, megaloblastic reaction in bone marrow along with giant metamyelocytes, mild dyserythropoiesis. Inconclusive cases were confirmed by serum Vitamin B12 and S. Folic acid level.Diagnosis of aplastic anaemia was made by marrow hypocellularity to acellularity in bone marrow aspirates..Thalassemia and other haemoglobinopathies were diagnosed from HPLC findings. Anaemia of chronic disease was diagnosed from history, increased marrow iron content and S. Ferritin, CRP in the light of the underlying disease.

Sideroblastic anaemia was diagnosed cumulatively from a dimorphic blood picture, ring sideroblasts in bone marrow and history of an underlying cause. Before labelling as sideroblastic anaemia, other causes of dimorphic anaemia were excluded.

Provisional diagnosis of MDS was made from a history of refractory anaemia, unilineage to multilineage dysplasia in bone marrow and presence of significant number of $(>15 \%)$ ring sideroblasts in bone marrow.

Iron store was graded according to the criteria laid down by Gale et al in 1963. Iron store was graded from 0 to
$6+$. Normal iron store was graded from $1+$ to $3+.0$ grade indicated absent iron store while grade $4+$ to $6+$ indicated increased iron store.

\section{Result}

Work up on 128 cases of anaemia led us to discover iron deficiency anaemia (IDA), megaloblastic anaemia (MA), aplastic anaemia (AA), thalassemia and other haemoglobinopathies , sideroblastic anaemia(SA), myelodysplastic anaemia(MDS) and anaemia of chronic disease(AOCD)) as causes of anaemia in adults.

In our study, maximum number of cases were in 20-30 years of age $(31.3 \%)$ and a minimum in elderly age group (71-80years) (6.25\%) [fig.1]Prevalence of anaemia was more in males (62\%). [fig.2]

Anaemia (49\%) was most common type of haematological presentation. [fig.3]. IDA was most common anaemia to present with anaemia only .Bicytopenia was main presenting feature of megaloblastic anaemia (MA) (61.5\%) while sideroblastic anaemia (SA) and aplastic anaemia (AA) presented with pancytopenia with equal (44.4\%) frequency. [tab.1]

Irrespective of initial haematological presentation, IDA was most common cause of anaemia with male preponderance. [tab.2]. Peak incidence was in 20-30 years of age with absent or low normal ( 0 to $1+$ ) iron store in bone marrow fragments.[tab.3]

Megaloblastic anaemia was second major cause of anaemia $(28.2 \%)$ with a female preponderance and presenting mainly with bicytopenia.[tab.2,tab.1] Young age (20-30 years) was the most common effected group $(55.5 \%)$ with a normal $(2+$ to $3+)$ iron store.[tab.3]

Least common finding was myelodysplastic syndrome (MDS) (0.8\%) followed by sideroblastic anaemia(SA) $(2.3 \%)$, both with a female preponderance. [tab.2] The only case of MDS was recorded in 61-70 years of age with increased(5+) iron store while SA was seen in 51-70 years of age with increased(4+ to $5+$ ) iron store.[tab.3]

Thalassemia and other haemoglobinopathies (sickle cell trait and disease, E-beta thalassemia trait) made up 12.5\% of total findings presenting mainly as anaemia followed by bicytopenia and pancytopenia. Majority of cases belonged to $20-30$ years of age $(75 \%)$ with male preponderance and found to have high normal to increased $(3+$ to $4+)$ iron content in bone marrow. [tab.3]

AA constituted $2.3 \%$ of all cases, all presented with pancytopenia with a male preponderance and an equal age 
distribution in most of the groups. All the cases had normal to mildly increased $(2+$ to $4+)$ marrow iron content. [tab.3]

Anaemia of chronic disease (AOCD) constituted $12.5 \%$ of total findings. AOCD was recorded in 41-70 years of age with male preponderance and presenting with anaemia and bicytopenia. They had high normal to increased (3+ to $5+$ ) marrow iron content. [tab:1,tab.3]

Perls' stain revealed presence of ring sideroblast in only $(n=4) 3.12 \%$ and few cases showed increased number of sideroblasts in bone marrow. A 62 years old lady with refractory anaemia not responding to therapy and with no systemic illness was referred for bone marrow aspiration analysis. Bone marrow was hypercellular, megaloblastoid erythropoiesis, and ring sideroblast was significantly increased. Exact enumeration of ring sideroblast was not possible due to diluted smear. These findings led to the diagnosis of the case as MDS (refractory anaemia with ring sideroblast, RARS) $(0.78 \%)$. 3 cases showed few ring sideroblasts and history of chronic alcoholism in 2 cases and antitubercular therapy (isoniazid) in one. On the basis of these findings and the history, these cases were labelled as secondary sideroblastic anaemia (2.3\%) due to alcoholism and antitubercular therapy.[tab.4]

Table 1: distribution of anaemias with initial haematological finding.

\begin{tabular}{|l|c|c|c|c|c|c|c|c|}
\hline cytopenia & IDA & MA & AA & THAL \& Others & SA & MDS & AOCD & Total \\
\hline Anaemia & 37 & 0 & 0 & 10 & 3 & 0 & 12 & 62 \\
\hline Bicytopenia & 7 & 24 & 0 & 4 & 0 & 0 & 4 & 39 \\
\hline pancytopenia & 0 & 12 & 12 & 2 & 0 & 1 & 0 & 27 \\
\hline
\end{tabular}

Table 2: sex distribution of different types of cases.

\begin{tabular}{|l|c|c|c|}
\hline anaemia & Male(\%) & Female(\%) & Total \\
\hline IDA & $32(72.7)$ & $12(27.3)$ & 44 \\
\hline MA & $16(44.4)$ & $20(55.6)$ & 36 \\
\hline AA & $8(66.6)$ & $4(33.3)$ & 12 \\
\hline Thalasemia \& other haemoglobinopathies & $12(75)$ & $4(25)$ & 16 \\
\hline SA & $1(33.3)$ & $2(66.7)$ & 3 \\
\hline MDS & 0 & $1(100)$ & 1 \\
\hline AOCD & $10(62.5)$ & $6(37.5)$ & 16 \\
\hline
\end{tabular}

Table.3: Iron content in marrow in different types of anaemias in various age groups:-

\begin{tabular}{|c|c|c|c|c|c|c|c|}
\hline Anaemia & $20-30$ yrs & $31-40$ yrs & $41-50$ yrs & $51-60$ yrs & $61-70$ yrs & $71-80$ yrs & Total cases \\
\hline IDA & $\begin{array}{c}8 \\
(0-1+) \\
\end{array}$ & $\begin{array}{c}20 \\
(0-1+)\end{array}$ & $\begin{array}{c}4 \\
(0) \\
\end{array}$ & $\begin{array}{c}4 \\
(0) \\
\end{array}$ & $\begin{array}{c}4 \\
(0) \\
\end{array}$ & $\begin{array}{c}4 \\
(0) \\
\end{array}$ & $\begin{array}{c}44 \\
(34.4 \%)\end{array}$ \\
\hline MA & $\begin{array}{c}20 \\
(2+-3+)\end{array}$ & $\begin{array}{c}4 \\
(2+-3+) \\
\end{array}$ & $\begin{array}{c}8 \\
(2+-3+) \\
\end{array}$ & $\begin{array}{c}4 \\
(2+-3+)\end{array}$ & 0 & 0 & $\begin{array}{c}36 \\
(28.1 \%)\end{array}$ \\
\hline AA & 0 & $\begin{array}{c}4 \\
(2+-3+)\end{array}$ & $\begin{array}{c}4 \\
(2+-4+)\end{array}$ & 0 & $\begin{array}{c}4 \\
(2+-3+)\end{array}$ & 0 & $\begin{array}{c}12 \\
(9.4 \%)\end{array}$ \\
\hline $\begin{array}{l}\text { Thalassemia } \\
\text { \& others }\end{array}$ & $\begin{array}{c}12 \\
(3+-4+)\end{array}$ & 0 & $\begin{array}{c}4 \\
(3+-4+)\end{array}$ & 0 & 0 & 0 & $\begin{array}{c}16 \\
(12.5 \%)\end{array}$ \\
\hline SA & 0 & 0 & 0 & $\begin{array}{c}2 \\
(4+-5+) \\
\end{array}$ & $\begin{array}{c}1 \\
(5+)\end{array}$ & 0 & $\begin{array}{c}3 \\
(2.3 \%)\end{array}$ \\
\hline MDS & 0 & 0 & 0 & 0 & $\begin{array}{c}1 \\
(5+)\end{array}$ & 0 & $\begin{array}{c}1 \\
(0.8 \%)\end{array}$ \\
\hline AOCD & 0 & 0 & $\begin{array}{c}4 \\
(4+-5+)\end{array}$ & $\begin{array}{c}6 \\
(3+-5+)\end{array}$ & $\begin{array}{c}2 \\
(4+-5+)\end{array}$ & 0 & $\begin{array}{c}16 \\
(12.5 \%)\end{array}$ \\
\hline
\end{tabular}

Table 4: presence of ring sideroblast in bone marrow aspirates.

\begin{tabular}{|l|c|c|}
\hline Ring sideroblast & Number of Cases(n) & $\%$ \\
\hline Present & 4 & 3.12 \\
\hline absent & 124 & 96.87 \\
\hline
\end{tabular}




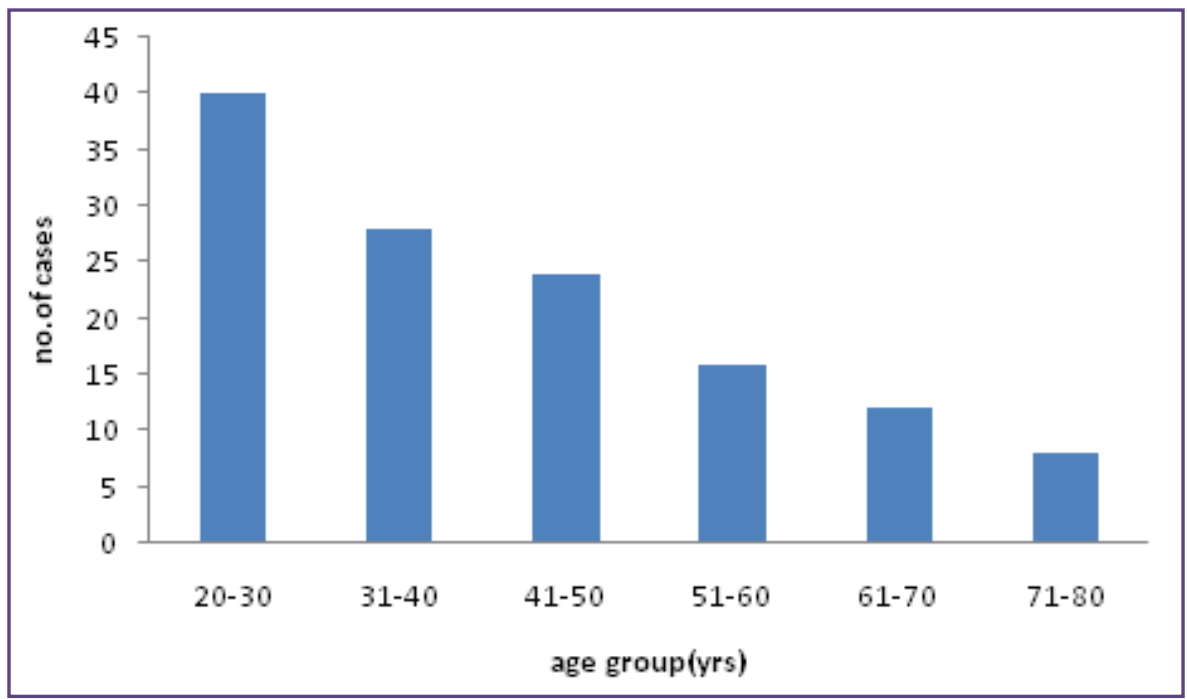

Fig. 1: age distribution of cases:

\section{$\%$ cases}

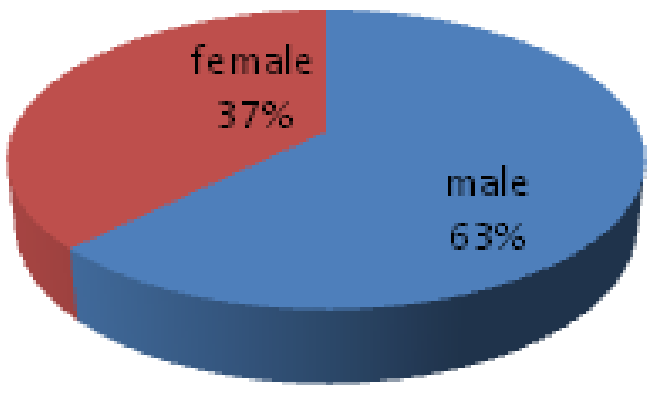

Fig. 2: sex distribution of total cases.

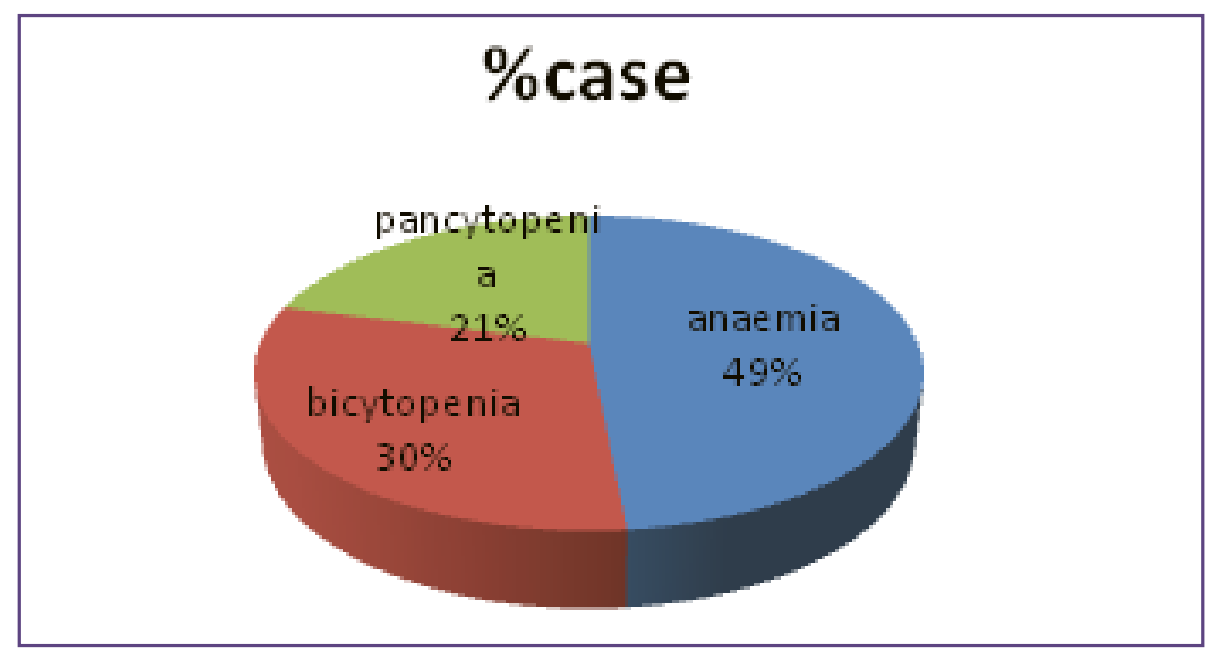

Fig. 3: cases (\%) according to initial haematological presentation. 


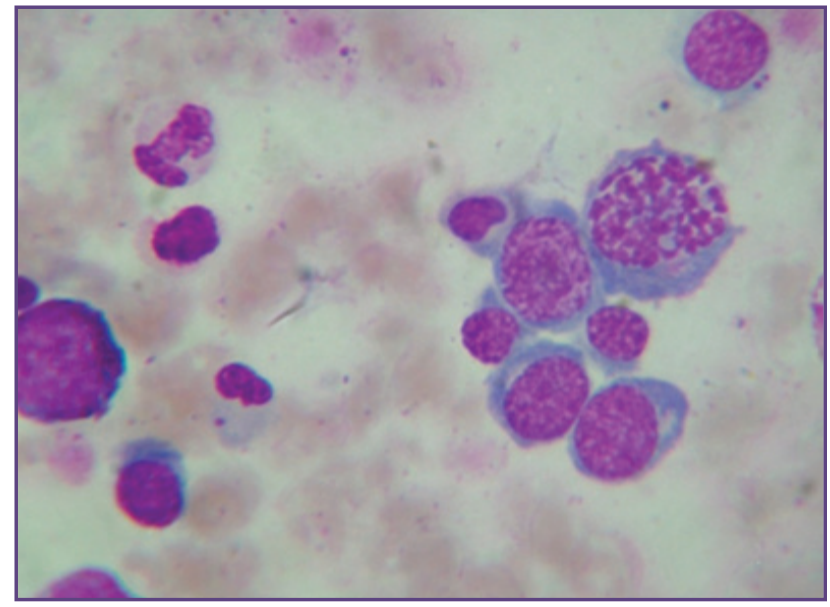

Fig. 4: Megaloblastoid reaction in bone marrow aspirate. Leishmen's stain.100X.

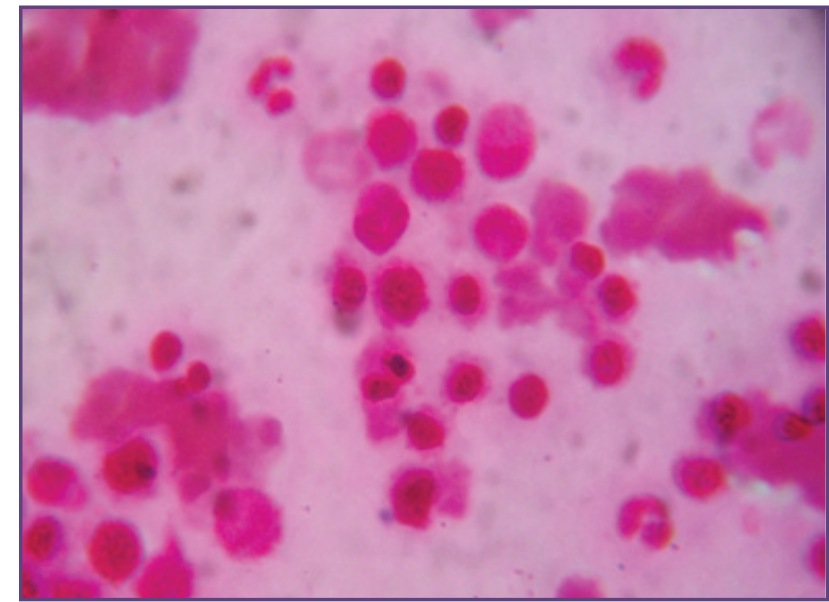

Fig. 5: Absence of sideroblasts in bone marrow aspirate in iron deficiency anaemia. 1\%Pararosalinine counterstain.40X.

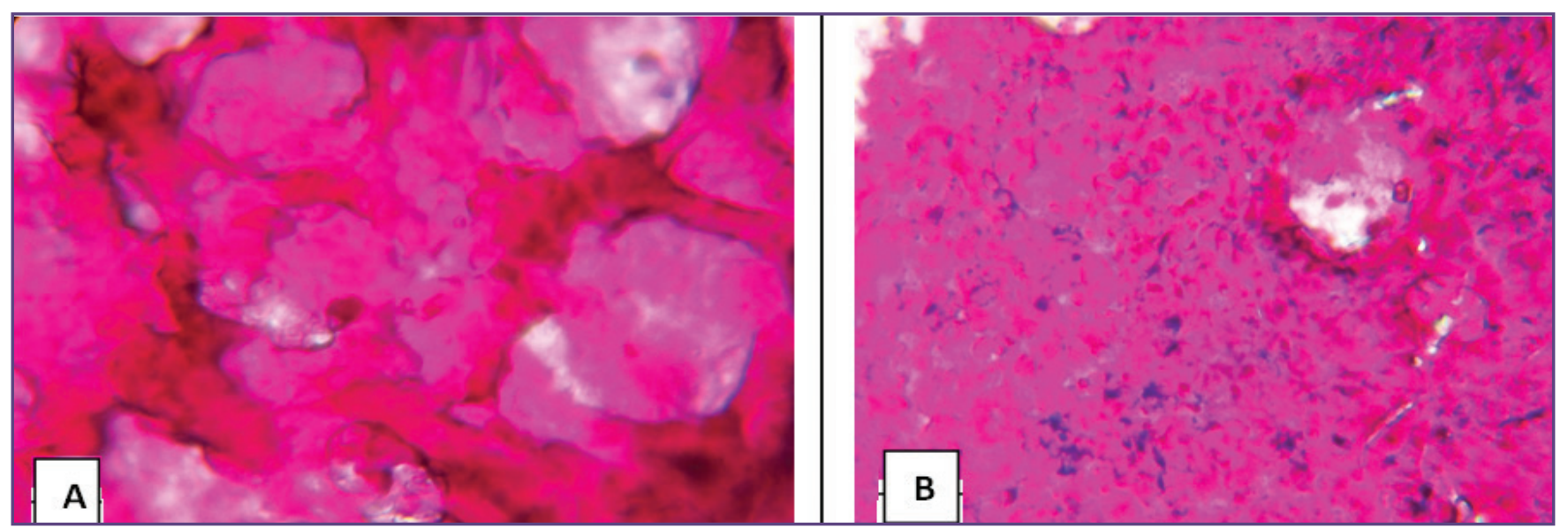

Fig. 6: Perl's stain in bone marrow aspirates.1\% Pararosalinine counterstain.(A) grade0 iron store.100X (B) grade2 iron store.40X

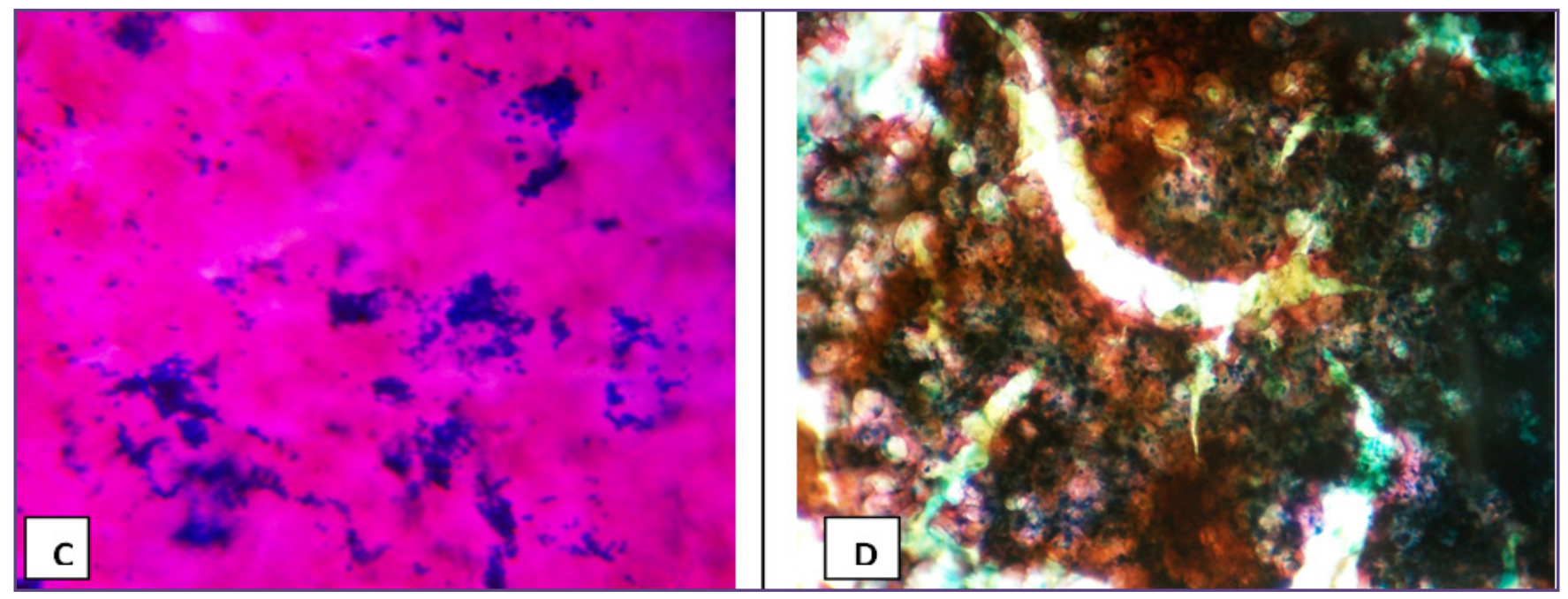

Fig. 7: Perl's stain in bone marrow aspirates.100X.(C) grade3 iron store.1\% Pararosalinine counterstain (D) grade6 iron store. $1 \%$ Neutral red counterstain. 


\section{Discussion}

Anaemia is commonly encountered sign and symptom in adults but indication for bone marrow aspirate examination was relatively less and cases were referred for the same only when non-invasive techniques could not give a definitive diagnosis.

In our study, maximum number of cases were in 20-30 years of age $(31.3 \%)$ followed by $31-40$ years $(21.3 \%)$ which was consistent with the finding in Pujara et al who also recorded $30 \%$ and $27 \%$ of cases in these age group respectively ${ }^{[5]}$ IDA was the most common finding in our study with a iron store of 0 to $1+$. This finding was similar to the finding in Pujara et al.MA was second most common finding recorded in our study with a normal iron store $(2+$ to $3+)$. While Pujara et al found increased store, Dharwadkar et al found normal (1+to $3+)$ iron store in bone marrow fragments. ${ }^{[5,6]}$ This dissimilarity with Pujara et al may be due to the grading followed by them who considered 1+ to $2+$ iron as normal store while grades above that was recorded as increased store.

AA in our study constituted $44.5 \%$ of cases of pancytopenia with a male preponderance. Our study showed a normal to mildly increased iron store of $2+$ to $4+$. Similar finding was noted in Halder et al who found AA to be the most common cause of pancytopenia(79\%) with a male preponderance. Their study recorded $78.95 \%$ cases with increased and $21.05 \%$ with decreased iron store. ${ }^{[7]}$ Pujara et al also found absent to low normal marrow iron content in the cases of aplastic anaemia. ${ }^{[5]}$ This dissimilarity may be due to lesser number of cases of AA recorded in our study. Increased iron store in AA may be due to associated autoimmune disorder which causes AA.

In our present study, out of the 16 cases, $50 \%$ had sickle cell trait , 43\% had beta-thalassemia trait ,6.25\% had E-beta thalassemia trait. They had $3+$ to $4+$ iron store. Kaduri et al in his review article mentioned that iron deficiency is more common in sickle cell disease. According to him, deficient iron state is beneficial as it reduces sickling by decreasing MCHC-S amount in RCBs. ${ }^{[8]}$ Oluboyede et al found iron deficiency in sickle cell anaemia to be due to increased urinary iron loss. ${ }^{[9]}$ Normal to increased iron content in our study may be due to transfusion in few of our cases of sickle cell anaemia. Corwin et al in their study found coincidence of thalassemia minor with homozygosity for haemochromatosis as a cause of iron overload in non transfused cases of thalassemia minor. ${ }^{[10]}$ E-beta thalassemia minor is a non transfusion dependent state and further evaluation could establish such coincidental finding which is beyond the scope in our setup.
In our study, cases of sideroblastic anaemia showed high normal to increased iron store which is consistent with already established fact.

In our study, it was observed that majority of cases $(62.5 \%)$ of AOCD had high normal and rest with increased marrow iron content. It was on contrary to the finding in Pereira et al who found majority of cases with diminished to absent iron content $(73.3 \%){ }^{[11]}$

In our study, $3.2 \%$ cases showed ring sideroblasts in their marrows. Of these cases, $0.78 \%$ had myelodysplastic syndrome (RARS) and 2.3\% had secondary siderolastic anaemia. Dharwadkar et al found $1.8 \%$ cases of refractory anaemia with $10 \%$ marrow sideroblast. ${ }^{[6]}$ This difference may be due to their study sample which included cases of neoplasia also. Andrew et al found $3.4 \%$ cases of MDS which included $2(1.14 \%)$ cases of RARS. ${ }^{[12]}$ Lower incidence in our study may be due to the wider age range in which the study had been conducted unlike the study of Andrew et al who carried out the study in age 65 years and above. Similar finding was recorded in Aul et al who recorded 3.2\% cases of MDS in their study and also concluded that with increasing age the incidence of MDS increases. ${ }^{[13]}$

\section{Conclusion}

Proper evaluation of anaemia in adults is important for proper management on failure of which may lead to fatal consequences in long run. In our study, it is seen that Perls' stain is a cheap and relatively simple test that can be used as a routine test in all bone marrow aspirates to provide provisional diagnosis of some relatively infrequent causes of chronic anaemias which otherwise could be missed when only non invasive methods are relied upon for assessing body iron content. However studies on using Perls' stain in broader spectrum of haematological disorders may lead to more conclusive outcome.

\section{Reference}

1. Worldwide prevalence of anaemia 1993-2005 : WHO global database on anaemia / Edited by Bruno de Benoist, Erin McLean, Ines Egli and Mary Cogswell.

2. Gale E, Torrance J, Bothwell T. The Quantitative Estimation of Total Iron Stores in Human Bone Marrow. Journal of clinical investigation 1963;42:1076.

3. Burns E, Goldberg SN, Lawrence C, Wenz B. Clinical utility of serum tests for iron deficiency in hospitalized patients. Am J Clin Pathol 1990;93:240-45.

4. Koca E, Cetiner DA, Buyukasik Y, et al. Bone Marrow Iron staining is a Reliable Test for Elimination of Iron Deficiency Anaemia Rather than its Diagnosis. Int J Haematol oncol 2013;23:260-63. 
5. Pujara KM, Bhalara RV, Dhruva GA. A study of bone marrow iron storage in haematological disorders. Int J Health Allied Sci 2014;3:221-24.

6. Dharwadkar A, Vimal S, Panicker Narayanan K, Chandanwale Shirish S, Vishwanathan V, Kumar H. Study of sideroblast and iron stores in bone marrow aspirates using Perls's stain. Medical journal of Dr. D.Y. Patil university 2016;9:181-85.

7. Haldar B, Partha PP, Sarkar TK, Sharma S, Goswami KB, Aikat A. Aplastic Anemia:A Common Hematological Abnormality Among Peripheral Pancytopenia. N Am J Med Sci 2012;4:384-88.

8. Kaduri P. R. Iron in Sickel cell disease: A review why less is better. Am. J Haemtol 2003;73:59-63.

9. Oluboyede OA, Ajayi OA, Adeyokunnu AA. Iron studies in patients with sickle cell disease. Afr J Med Med Sci 1981;10:1-7.
10. Edwars CQ, Skolnick MH, Kushnew JP. Coincidental nontransfusional iron overload and thalassemia minor: Association with HLA-Linked Haemochromatosis. Blood $1981 ; 58: 844-48$

11. Pereira M R M, Veloso E R P, Menezes Y, Gualandro S, Vassalo J Yosinaria. Bone Marrow Finding In Systemic Lupus Erythematosus Patients With Peripheral Cytopenias. Clinical Rheumatol 1998;17:219-22.

12. Artz AS, Thirman MJ. Unexplained Anemia Predominates Despite an Intensive Evaluation in a Racially Diverse Cohort of Older Adults From a Referral Anemia Clinic. J Gerontol A Biol Sci Med Sci 2011;66:925-32.

13. Aul C, Gattermann N, Schneider W. Age related incidence and other epidemiological aspect of myelodysplastic syndromes. British journal of Haemtol 1992;8:358-67.

*Corresponding author:

Dr. Riju Rani Deka, C/O P. C. Deka,Bishnu Nagar, Bishnu Rabha Path, P.O- Tezpur.Dist-Sonitpur,Assam, Pin-784001, India

Phone: +91 9435180795

Email: dr.rijurdeka82@gmail.com

Date of Submission : 09.08.2016

Date of Acceptance : 10.04.2017

Financial or other Competing Interests: None.

Date of Publication : 31.08.2017 